\title{
Predicting response to radical (chemo)radiotherapy with circulating HPV DNA in locally advanced head and neck squamous carcinoma
}

Jen Y Lee ${ }^{1}$, Isaac Garcia-Murillas ${ }^{1}$, Rosalind J Cutts ${ }^{1}$, David Gonzalez De Castro ${ }^{2,3}$, Lorna Grove ${ }^{2}$, Tara Hurley ${ }^{2}$, Fuqiang Wang ${ }^{2}$, Christopher Nutting ${ }^{1,2}$, Katie Newbold ${ }^{1,2}$, Kevin Harrington ${ }^{1,2}$, Nicholas Turner ${ }^{1,2}$ and Shreerang Bhide ${ }^{\star, 1,2}$

${ }^{1}$ The Institute of Cancer Research, Fulham Road, London SW3 6JB, UK and ${ }^{2}$ The Royal Marsden Hospital, Fulham Road, London SW3 6JJ and Downs Road Sutton, SM2 5PT, UK

Background: Following chemo-radiotherapy (CCRT) for human papilloma virus positive (HPV +) locally advanced head and neck cancer, patients frequently undergo unnecessary neck dissection (ND) and/or repeated biopsies for abnormal PET-CT, which causes significant morbidity. We assessed the role of circulating HPV DNA in identifying 'true' residual disease.

Methods: We prospectively recruited test $(n=55)$ and validation $(n=33)$ cohorts. HPV status was confirmed by E7 RT-PCR. We developed a novel amplicon-based next generation sequencing assay (HPV16-detect) to detect circulating HPV DNA. Circulating HPV DNA levels post-CCRT were correlated to disease response (PET-CT).

Results: In pre-CCRT plasma, HPV-detect demonstrated 100\% sensitivity and $93 \%$ specificity, and $90 \%$ sensitivity and $100 \%$ specificity for the test $(27 \mathrm{HPV}+)$ and validation $(20 \mathrm{HPV}+$ ) cohorts, respectively. Thirty-six out of 37 patients (test and validation cohort) with complete samples-set had negative HPV-detect at end of treatment. Six patients underwent ND (3) and repeat primary site biopsies (3) for positive PET-CT but had no viable tumour. One patient had positive HPV-detect and positive PET-CT and liver biopsy, indicating 100\% agreement for HPV-detect and residual cancer.

Conclusions: We demonstrate that HPV16-detect is a highly sensitive and specific test for identification of HPV DNA in plasma at diagnosis. HPV DNA post-treatment correlates with clinical response.

Organ-preserving, concomitant, cisplatin-based chemoradiotherapy (CCRT), followed by surgical salvage if required, is a standardof-care for locally advanced (stage III/IV disease) head and neck cancer (LAHNC) of the oropharynx, larynx and hypopharynx (Dillon and Harrington, 2015). Following radical CCRT, decisions regarding salvage surgery for residual primary and cervical lymph node disease are based on clinical examination and imaging.
${ }^{18}$ F-FDG PET-CT, when available, is the imaging modality of choice (Mehanna et al, 2016), with a negative predictive value in excess of 90\% (Abgral et al, 2009; Mehanna et al, 2016). However, the positive predictive value is sub-optimal and patients frequently undergo unnecessary neck dissection (ND; Hamoir et al, 2012) and/or repeated biopsies from the purportedly residual primary tumour. ND causes significant morbidity (fibrosis, facial/

\footnotetext{
*Correspondence: Dr S Bhide; E-mail: shreerang.bhide@icr.ac.uk

${ }^{3}$ Current address: Queens University Belfast, School of Medicine, Dentistry and Biomedical Sciences, 97 Lisburn Road, Belfast BT9 7BL, UK.
}

Received 20 April 2017; revised 26 June 2017; accepted 13 July 2017; published online 15 August 2017

(C) 2017 Cancer Research UK. All rights reserved 0007-0920/17 
hypoglossal nerve damage, reduced shoulder movement and swallowing problems) and repeated biopsies to rule out residual cancer at the primary site can lead to delayed mucosal healing and significant patient anxiety. Therefore, optimised predictors of residual disease are required as a means of guiding management decisions.

A proportion of LAHNC are causally related to the human papilloma virus (HPV; Schache et al, 2016). In the tumour, HPV DNA is integrated into the host genome or is present in episomal form (Hopman et al, 2004) and can be released in to the bloodstream. Circulating HPV DNA can potentially be used as a detection marker for HPV-related LAHNC. The utility of circulating HPV DNA in monitoring disease response following radical chemo-radiotherapy in LAHNC has not been extensively evaluated. In a retrospective study, Wang et al (2015) measured circulating tumour DNA in of 93 patients with head and neck cancer at various sub-sites 21 of whom had HPV positive $(\mathrm{HPV}+)$ LAHNC. Very limited correlative analyses were performed between circulating DNA and treatment response.

Here, we prospectively collected plasma DNA in a test and independent validation cohorts of homogeneous group of patients with LAHNC treated with primary chemo-radiotherapy. We developed an ultra-sensitive HPV DNA next generation sequencing (NGS) assay 'HPV16-detect', with the ability to detect HPV DNA. We demonstrate that HPV16-detect analysis in the posttreatment samples in test and validation cohorts correlates with clinical response following curative treatment for LAHNC, and can anticipate residual disease.

\section{MATERIALS AND METHODS}

Informed consent was obtained from all eligible patients with stage III/IV (AJCC 2007) squamous cell carcinoma of the oropharynx, larynx or hypopharynx. Institutional board (ref. no. CCR 4157) and ethics committee (ref. no. 14/NE/1055) approved the study. Patients $<71$ years old received CCRT (cisplatin, $100 \mathrm{mg} \mathrm{m}^{-2}$ days 1 and 29; Wilkins et al, 2013). Patients staged as having T4 and/or $\mathrm{N} 2 \mathrm{C} / \mathrm{N} 3$ disease and deemed medically fit received induction chemotherapy, IC (2 cycles of taxotere $\left(75 \mathrm{mg} \mathrm{m}^{-2}\right.$, cisplatin $75 \mathrm{mg} \mathrm{m}^{-2}, \mathrm{~d} 1$ and 5-fluorouracil $\left.750 \mathrm{mg} \mathrm{m}^{-2} \mathrm{~d} 1-5\right)$ followed by CCRT (Blanchard et al, 2013). Radiotherapy was delivered using a simultaneous integrated boost-IMRT technique as described previously (Bhide et al, 2007). Clinical target volume (CTV) 1 included primary tumour and involved lymph node groups. CTV2 included areas at risk of harbouring microscopic disease and elective lymph node groups. All patients were treated with doses of $65 \mathrm{~Gy}$ in 30 fractions $(2.17 \mathrm{~Gy} /$ fraction) over 6 weeks to the primary target. Doses to the elective target were $54 \mathrm{~Gy}$ in 30 fractions $(1.8 \mathrm{~Gy} /$ fraction) over 6 weeks. A single observer delineated gross tumour volumes for primary disease and lymp nodes based on pre-treatment ${ }^{18}$ F-FDG PET-CT and MRI.

At 12 weeks, response following treatment was assessed by clinical examination and ${ }^{18}$ F-FDG PET-CT. Patients with metabolically active lymph node disease alone on ${ }^{18} \mathrm{~F}$-FDG PET-CT underwent ND. Residual or equivocal uptake at primary and distant sites was confirmed with a biopsy.

Validation samples were obtained from patients in an independent prospective observational clinical trial of imaging in LAHNC (CCR no. 3926, Ethics ref. no. 13/LO/0067). The patients (Supplementary Table S1) had to fulfil the same eligibility criteria, underwent treatment and response assessment as described above.

Serial plasma samples were collected were collected at baseline (before IC or CCRT), pre-CCRT (in patients receiving IC), 6 weeks and 12 weeks following completion of treatment and at the time of further ${ }^{18}$ F-FDG PET-CT scan if indicated.
Table 1. Patient and tumour characteristics for the test cohort

\begin{tabular}{|l|c|}
\hline Variable & Number of cases \\
\hline Number of patients & 55 \\
\hline T-stage & 6 \\
1 & 25 \\
2 & 15 \\
3 & 9 \\
4 & \\
\hline N-stage & 7 \\
0 & 6 \\
1 & 2 \\
2 A & 28 \\
$2 B$ & 12 \\
2 C & \\
\hline Treatment & 12 \\
IC+CCRT & 43 \\
CCRT & \\
\hline Pack-years (includes ex smokers) & 15 \\
Non-smoker & 11 \\
$\leqslant 10$ & 29 \\
$>10$ & \\
\hline Tumour sub-site & 47 \\
Oropharynx & 4 \\
Larynx & 4 \\
Hypopharynx & \\
\hline Abbreviations: IC=induction chemotherapy; CCRT = cisplatin-based chemoradiotherapy. \\
\hline
\end{tabular}

Blood samples: plasma processing and DNA extraction. $20 \mathrm{ml}$ of blood was centrifuged at 1500 r.p.m. for 10 min within $3 \mathrm{~h}$ of collection. Plasma was isolated and frozen at $-80{ }^{\circ} \mathrm{C}$. Prior to extraction, plasma samples were further centrifuged at 14000 r.p.m. for $10 \mathrm{~min}$ at $4{ }^{\circ} \mathrm{C}$. DNA was extracted from $5 \mathrm{ml}$ of plasma using the QIAamp Circulating Nucleic Acid Kit (Qiagen, Hilden, Germany) according manufacturer's instructions. DNA was eluted in $50 \mu \mathrm{l}$ of AVE buffer and stored at $-20^{\circ} \mathrm{C}$. For the validation cohort, circulating DNA was extracted from $2 \mathrm{ml}$ of plasma using the MagMAX Cell-free DNA Isolation kit (ThermoFisher Scientific, Waltham, MS, USA) on a KingFisher Flex System (ThermoFisher Scientific) according to the manufacturer's instructions. The resulting DNA was eluted in $50 \mu \mathrm{l}$ of MagMAX Cell-free DNA Elution Solution. Plasma DNA was quantified using a Bio-Rad QX200 ddPCR system- using ribonuclease $\mathrm{P}$ as a reference gene as previously described (Garcia-Murillas et al, 2015).

Tumour biopsies: HPV16 detection in tumour. Formalin fixed paraffin embedded tumour blocks of the diagnostic biopsy samples were obtained. Ten $10 \mu \mathrm{m}$ unstained slides and two haematoxylin and eosin stained slides were obtained from representative FFPE blocks. Experienced pathologists assessed tumour content and cellularity and suitable areas of tumour were marked for macrodissection, if necessary. RNA was extracted using the RecoverAll Total Nucleic Acid isolation kit for FFPE (Thermo Fisher, Waltham, MS, USA) followed by cDNA synthesis using the High-Capacity cDNA Reverse Transcriptase kit (Thermo Fisher). Evidence of HPV integration was assessed by detection of E7 expression using methods and primers described previously (Lamarcq et al, 2002) in a 7500 Sequence detection system (Applied Biosystems, Foster City, CA, USA/Thermo Fisher). Any specimen producing a $\mathrm{dCt}<13$ was consider positive for HPV integration.

P16 status of tumours was confirmed using immunohistochemistry (details Supplementary Section). All immunohistochemical interpretations were made by consultant head and neck histopathologists. Diffuse strong nuclear expression of $>70 \%$ of tumour nuclei for p16 was considered positive. 
A
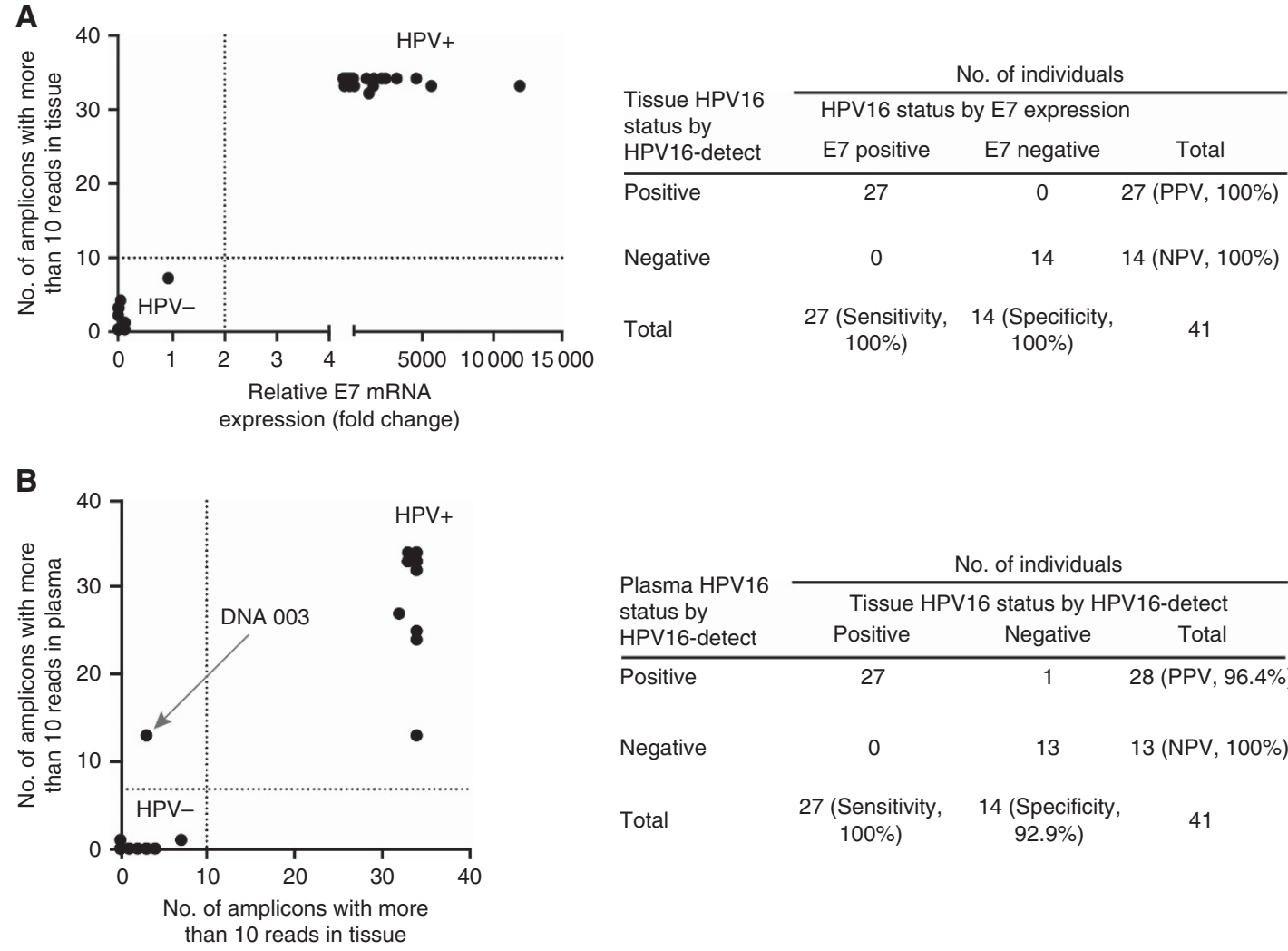

\begin{tabular}{lccc}
\multirow{2}{*}{$\begin{array}{l}\text { Plasma HPV16 } \\
\text { status by } \\
\text { HPV16-detect }\end{array}$} & $\begin{array}{c}\text { No. of individuals } \\
\text { Positive HPV16 status by HPV16-detect } \\
\text { Positive }\end{array}$ & Negative & Total \\
\hline Negative & 27 & 1 & 28 (PPV, 96.4\%) \\
Total & 0 & 13 & 13 (NPV, 100\%) \\
& 27 (Sensitivity, & 14 (Specificity, \\
100\%) & $92.9 \%)$ & 41
\end{tabular}



\begin{tabular}{lccc} 
& \multicolumn{3}{c}{ No. of individuals } \\
\cline { 2 - 4 } $\begin{array}{l}\text { Plasma HPV16 } \\
\text { status by }\end{array}$ & \multicolumn{2}{c}{ Tissue HPV16 status by p16-staining } \\
HPV16-detect & p16 Positive & p16 Negative & Total \\
\hline Positive & 18 & 0 & 18 (PPV, 100\%) \\
& & & \\
Negative & 2 & 13 & 15 (NPV, 86.67\%)
\end{tabular}

Total 20 (Sensitivity, 13 (Specificity, 33

Figure 1. Comparison of HPV16 status by E7 mRNA, p16 staining and HPV16-detect (NGS) assay in tissue and plasma. (A) There was $100 \%$ agreement between the HPV16 status assigned by the 'gold standard' E7 mRNA expression and HPV16-detect. (B) Test cohort: HPV16-detect assay demonstrated $100 \%$ sensitivity and $92.9 \%$ specificity in detecting HPV DNA in plasma compared to tissue. (C) Validation cohort: HPV16detect assay demonstrated $90 \%$ sensitivity and $100 \%$ specificity in assigning HPV status in plasma compared to p16 staining in tissue.

$\mathrm{NPV}=$ negative predictive value; $\mathrm{PPV}=$ positive predictive value.

HPV16 DNA sequencing in tumour and plasma (HPV16-detect assay design). To detect HPV DNA in patients we designed 'HPV16-detect' a novel NGS assay using Ion Ampliseq Designer (ThermoFisher Scientific). This comprised of a 39-amplicon single pool panel covering 34 distinct regions of the HPV16 genome, covering nucleotide variations in the most prevalent HPV16 subtypes (Burk et al, 2013; Cullen et al, 2015) and human reference genes (5 amplicons). Seven of the most prevalent strains of HPV16 (Burk et al, 2013; Cullen et al, 2015) were aligned using CLUSTAL O (1.2.1) multiple sequence alignment programme (ebi.ac.uk). Common regions surrounding base differences between the different strains were used to guide the design of the panel. Human sequences (exon 8 of GAPDH and exon 4 of ACTINB) were also included in the panel design as controls for library preparation and sequencing efficiency. Ion torrent libraries were prepared using Ion Ampliseq library preparation kit 2.0 (ThermoFisher Scientific) according to manufacturer's instructions using $5 \mathrm{ng}$ of tissue DNA or $3 \mathrm{ng}$ of plasma DNA. Plasma DNA was quantified as per the method detailed in the Supplementary Section. The barcoded libraries were quantified using the Ion Library TaqMan Quantitation kit before dilution to $100 \mathrm{pM}$ and pooling. Libraries were templated using the Proton Hi-Q templating kit on the Ion Chef and sequenced using a Proton PI Chip v2 and 520 flows. The average amplicon length was 136 bases and the genome size was $8964 \mathrm{bp}$ (including the human amplicons). The sequencing ranged between $\sim 100000-$ 14000000 reads per sample.

The sequencing data were aligned using the TorrentCaller aligner TMAP to an amalgamated HPV16 genome (accession no. K02718) with the human exons included. Bedtools v2.23.0 (Quinlan and Hall, 2010) was used to extract on-target reads with a minimum overlap of $50 \%$ with amplicons in the panel. Additionally reads with a mapping quality of $<15$ were removed using samtools v1.2 (Li et al, 2009).

From the cleaned bam files, coverage was calculated for each amplicon in the panel using coverage Bed. Reads were split into 

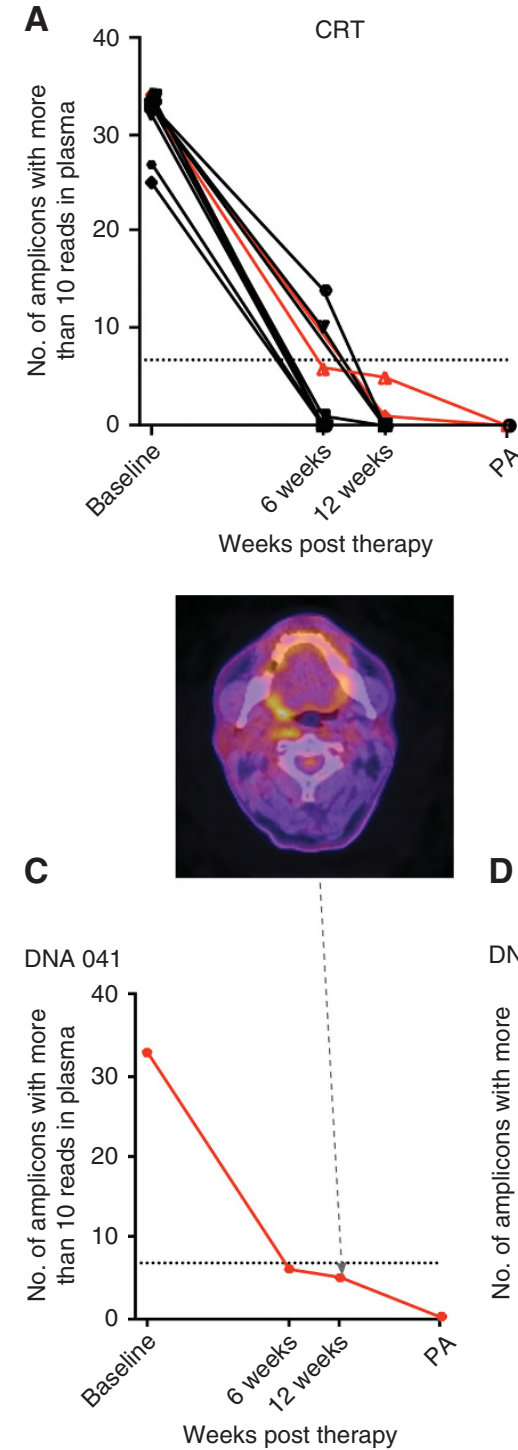
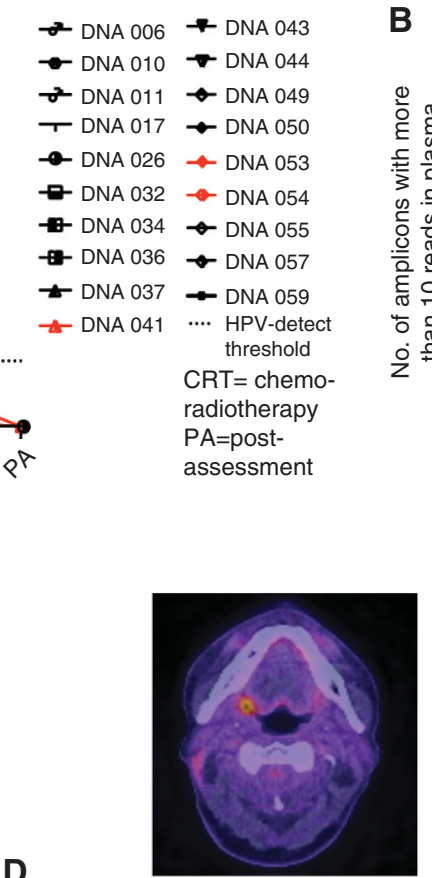

D

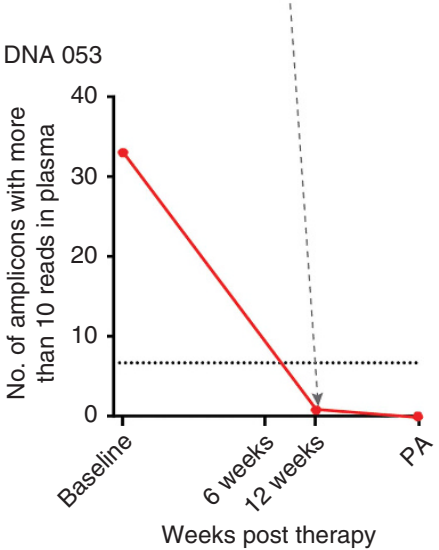

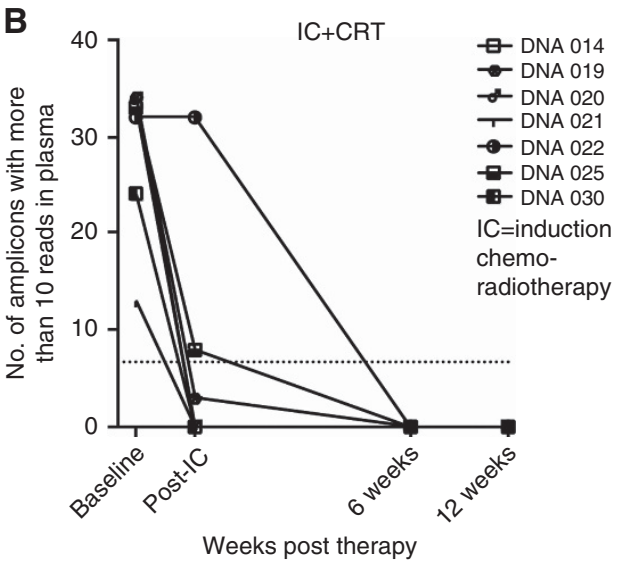

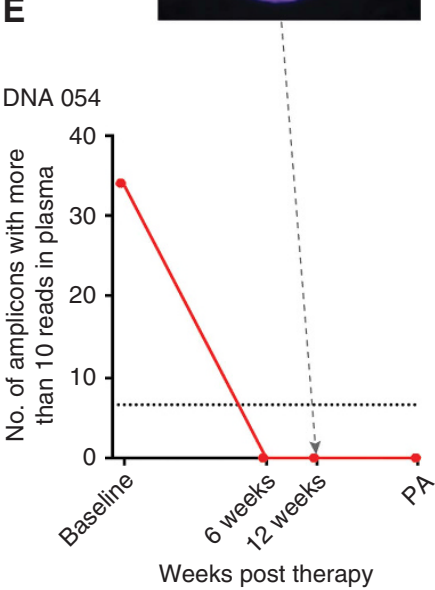

Figure 2. HPV DNA tracking in treatment responsive HPV + patients using the HPV16-detect assay. HPV DNA levels decreased below threshold at 12-weeks post-treatment in patients receiving C-CRT $(n=19, \mathbf{A})$ and in patients who received induction chemotherapy $(I C)$ and CRT $(n=7, B)$. Red plots in (A) highlight the three patients in (C-E). Residual disease was evident in three patients by ${ }^{18} \mathrm{~F}-\mathrm{FDG}$ PET-CT (C-E), with negative biopsies and below threshold levels of HPV DNA in the plasma taken at the time of biopsies (PA). PA=post-assessment; post-IC = post-induction chemotherapy. A full colour version of this figure is available at the British Journal of Cancer journal online.

those covering human and those covering HPV amplicons and coverage of each portion calculated individually.

To validate NGS detection of HPV DNA with HPV16-detect, we compared NGS HPV16 detection with E7 mRNA detection by RTPCR, considered as the 'gold-standard' assay for biologically relevant HPV infection (Schache et al, 2011). Baseline and post-CCRT plasma samples from HPV16 + patients in the test cohort were then sequenced using HPV16-detect. Presence of plasma HPV DNA was correlated to disease response following treatment. A similar methodology was used for the independent validation cohort, except for tumour HPV assignment, which based on P16 ${ }^{\mathrm{INK} 4 \mathrm{~A}}$ alone, to mirror current clinical practice. Analysis of plasma HPV DNA was performed blind to the patients HPV status and disease response.

Statistical analyses. Correlation analysis was used to quantify the relationship between: plasma HPV DNA levels at baseline with tumour volumes. Comparisons between different HPV16 assays in different tissue types were analysed using contingency diagrams and Fisher's exact test. All statistical analyses were calculated in GraphPad Prism version 6.
In order to classify HPV + and HPV- samples using HPV16detect in tissue, we set a threshold whereby a sample was classified positive if there were 10 reads present from more than 10 different HPV16 amplicons. For the experimental cohort, human reads ranged between 6000-72 000 and 57000-3000 000 for tissue and plasma, respectively. Thus, a minimum threshold of 50000 reads in the human amplicons was set for the plasma validation cohort to account for library preparation and sequencing efficiency. In order to assess the threshold for the number of amplicons needed for positive HPV16-detect readout in plasma-a ROC analyses was used. In the first step, HPV status was assigned using the gold standard E7 mRNA to separate the two groups. The number of amplicons with $>10$ reads at baseline was inputted for each patient to find a suitable threshold for this parameter. Sorting the values in both HPV positive and negative groups and averaging adjacent values in the sorted list generated a list of thresholds. Based on the ROC analyses a threshold of 10 reads in 7 amplicons gave the greatest sensitivity and specificity and was selected as threshold for classification of plasma as HPV DNA positive Supplementary Figure S2 and Supplementary Table S2). 

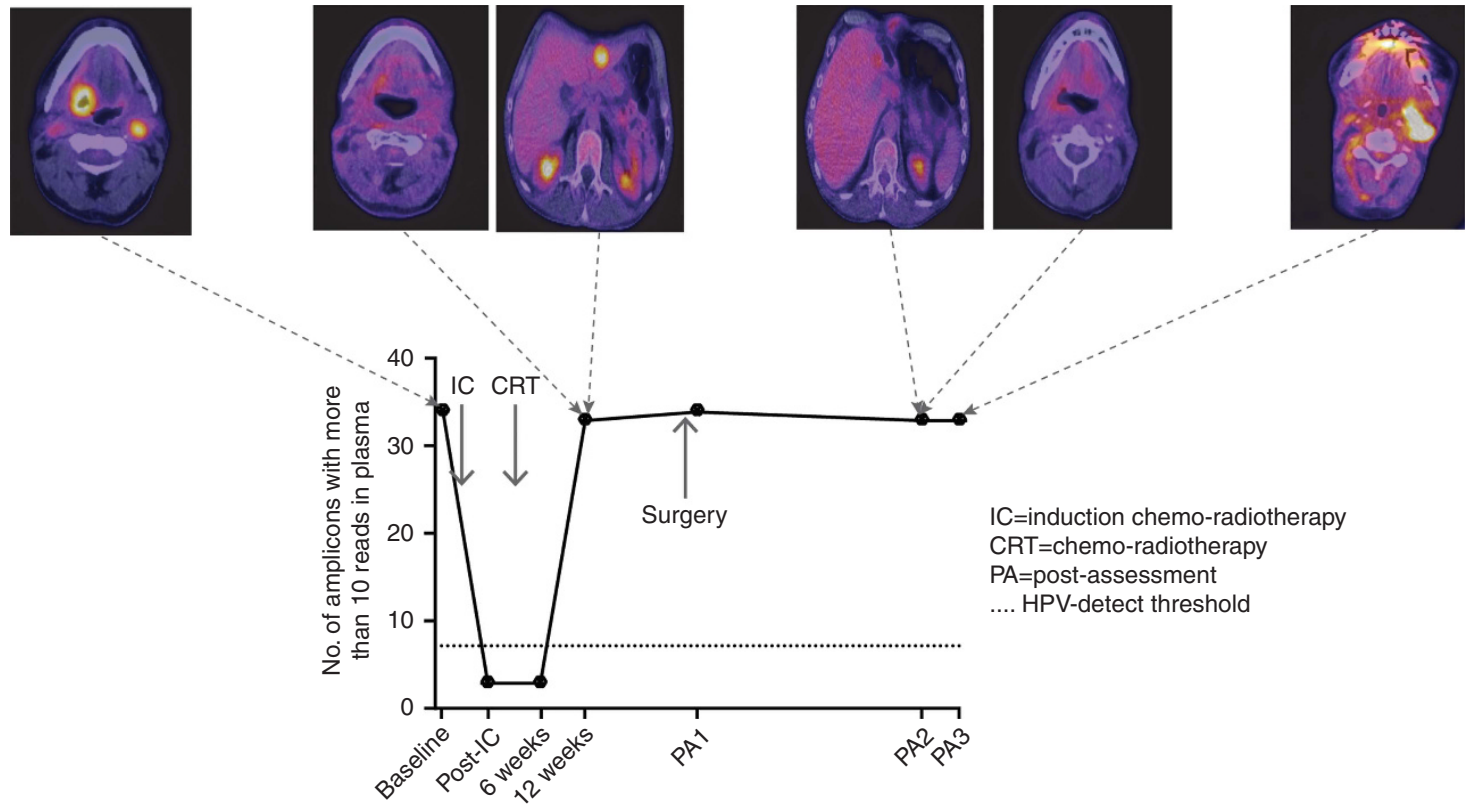

Weeks post therapy

Figure 3. HPV16-detect assay tracked presence of residual disease with ${ }^{18} \mathrm{~F}$-FDG PET-CT at corresponding time points. HPV DNA levels decreased in response to treatment at 6 weeks, but increased significantly at 12 weeks, which corresponded to presence of liver metastases on ${ }^{18}$ F-FDG PET-CT. HPV DNA levels decreased post-liver resection, however never dropped below the threshold. ${ }^{18} \mathrm{~F}-\mathrm{FDG}$ PET-CT 8 months following liver surgery revealed disease relapse in cervical lymph nodes. CRT = chemo-radiotherapy; PA = post-assessment; post-IC $=$ postinduction chemotherapy. A full colour version of this figure is available at the British Journal of Cancer journal online.

\section{RESULTS}

Fifty-nine patients were recruited into the study. Details for 55 (4 withdrew consent) patients are shown in Table 1. Tumour blocks were not available in five patients. Adequate DNA and RNA could be extracted from the tumour blocks of $42 / 50$ patients. Twentyseven samples were positive for E7 (and $\mathrm{p} 16^{\mathrm{fNK} 4 \mathrm{~A}}$ ) and these patients were classed as HPV + . All of these patients had cancers of the oropharynx.

Validation of HPV16-detect. Detection of HPV DNA with HPV16-detect was correlated with E7 mRNA detection for validation and demonstrated $100 \%$ specificity (95\% confidence interval (CI) 76.84-100\%) and sensitivity (95\% CI 87.23-100\%; $P<0.0001$, Figure 1A).

Sequencing of baseline plasma samples from the $27 \mathrm{HPV}+$ patients using HPV16-detect demonstrated sensitivity and specificity of $100 \%(95 \%$ CI $87.23-100 \%)$ and $92.9 \%$ respectively (95\% CI 66.13-99.82\%), $(P<0.0001$, Figure 1B). HPV DNA was not detected in any of the thirteen patients with HPV- tumours, who served as a negative control (Supplementary Figure S1). There was no correlation between the volumes of disease (primary and lymph nodes) and HPV DNA levels in plasma (Supplementary Table S3).

The validation cohort had 33 patients (Supplementary Table S1). Using HPV16-detect, HPV DNA was detected in the baseline plasma of 18 of the 20 patients HPV + patients (on p16 ${ }^{\mathrm{INK} 4 \mathrm{~A}} \mathrm{IHC}$ ) but not in negative controls (HPV-patient samples; Figure 1C and Supplementary Figure S1). This gave a sensitivity and specificity of 90\% (95\% CI $68.30-98.77 \%)$ and $100 \%$, (95\% CI $75.29-100 \%)$ respectively $(P<0.0001$, Fisher's exact test, Figure $1 \mathrm{C})$.

HPV DNA tracking for HPV + tumours to identify residual disease. Twenty-three (including the two HPV + patients with PIK3CA mutation) of the $27 \mathrm{HPV}+$ patients had complete radiological response as assessed by ${ }^{18}$ F-FDG PET-CT at 12 weeks post-CCRT. HPV DNA levels were below the threshold of detection in all 23 patients (Figure 2A and B). Three of the four remaining patients had increased ${ }^{18} \mathrm{~F}$-FDG uptake corresponding to the primary site (Figure 2C-E). Biopsies from the PET-avid sites failed to demonstrate residual tumour. Further ${ }^{18}$ F-FDG PET-CT scans demonstrated complete resolution of disease. HPV DNA levels remained below the threshold of detection at all time points.

In one patient ${ }^{18} \mathrm{~F}$-FDG PET-CT demonstrated increased $18 \mathrm{~F}$ FDG uptake in the liver, but no active disease at the primary site or cervical lymph nodes with elevated HPV DNA levels (Figure 3). The single biopsy-proven $(\mathrm{HPV}+$ ) liver lesion was surgically resected, resulting only in a marginal fall in plasma HPV DNA levels. Further ${ }^{18}$ F-FDG PET-CT failed to demonstrate any disease. However, plasma HPV DNA levels showed a continued rise and ${ }^{18}$ F-FDG PET-CT, performed 8 months following liver resection, demonstrated increased uptake in the cervical lymph nodes. The presence of recurrent neck disease was confirmed at ND.

One patient with $\mathrm{p} 16^{\mathrm{INK} 4 \mathrm{~A}}$ IHC-positive tumour with inadequate tumour RNA for E7 analysis (therefore not included in the $27 \mathrm{HPV}+$ patient cohort) had increased ${ }^{18} \mathrm{~F}$-FDG PET-CT uptake in a level II node and underwent ND, which failed to show any viable tumour. HPV DNA was detectable in baseline plasma but undetectable in plasma sample prior to ND.

Ten of the twenty HPV + patients in the validation cohort had complete sets of serial plasma samples. HPV DNA was not detected at 12 weeks post-CCRT in any of these 10 patients (Figure 4A). Seven these patients had complete radiological response on ${ }^{18}$ F-FDG PET-CT. Three patients with increased ${ }^{18} \mathrm{~F}-\mathrm{FDG}$ uptake in cervical lymph nodes underwent ND and, as with the patient in the test cohort, the specimen showed no evidence of viable tumour (Figure 4B-D).

\section{DISCUSSION}

We describe 'HPV16-detect', a novel ultra-sensitive method of detecting and tracking HPV DNA in HPV + LAHNC in a study with prospective collection of biological samples and detailed 

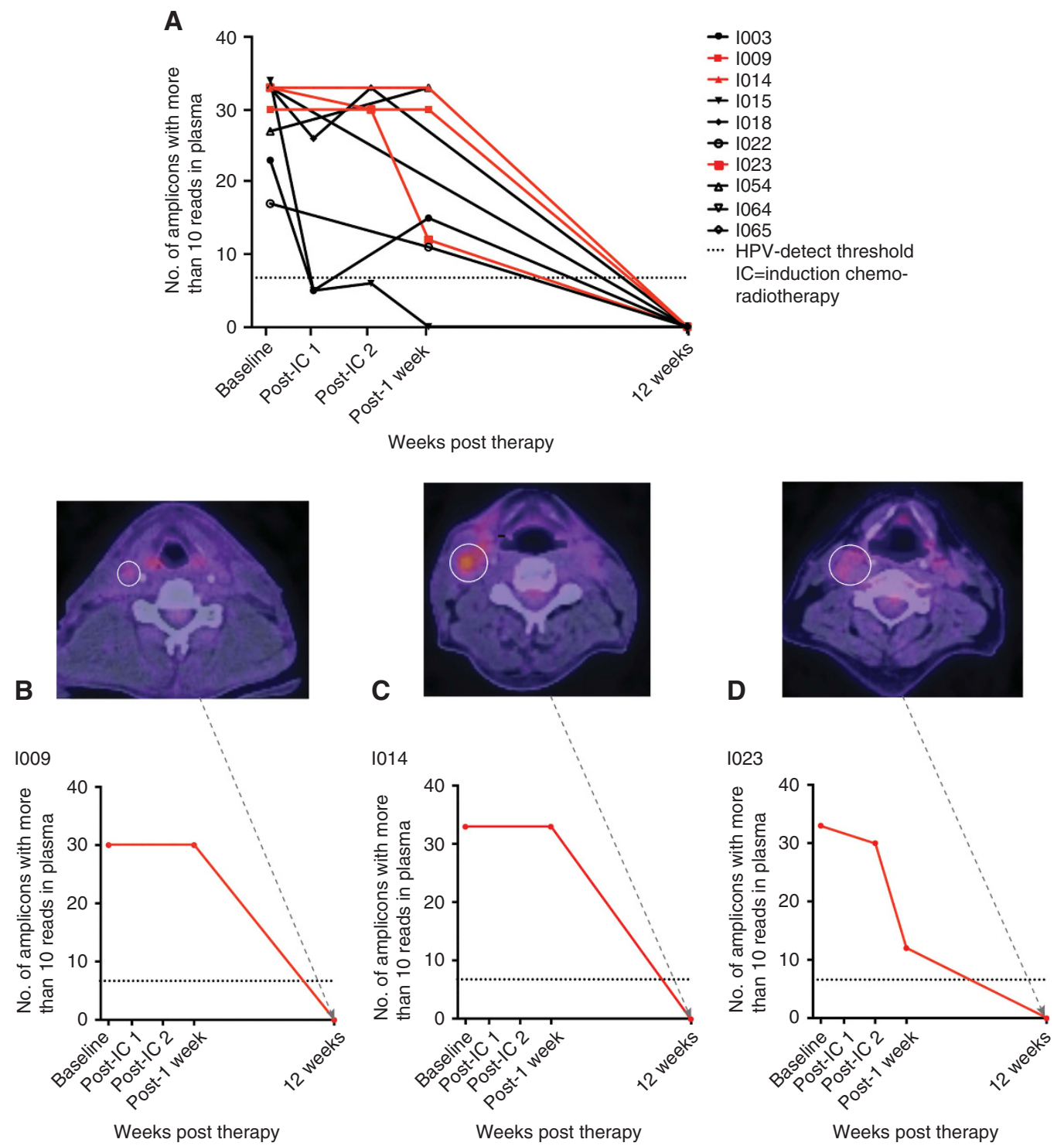

Figure 4. HPV DNA tracking in the validation cohort with HPV16-detect assay. HPV DNA levels decreased below threshold at 12-weeks posttreatment $(n=10, \mathbf{A})$. Red plots in (A) highlight the three patients in (B-D) with residual lymph node disease on ${ }^{18} \mathrm{~F}-\mathrm{FDG}$ PET-CT, negative ND specimens and below threshold levels of HPV DNA in the plasma taken at the time ND. PA= post-assessment; post-IC = post-induction chemotherapy; Post-1 week= post-5 fractions of radiotherapy. A full colour version of this figure is available at British Journal of Cancer journal online.

clinical data. Amplicon-based sequencing of multiple regions of the viral genome enabled us to detect HPV DNA at baseline with high sensitivity and specificity. Tracking HPV DNA in sequential samples through and after chemo-radiotherapy predicted response and residual disease at the primary site and in cervical lymph nodes suggesting the potential of HPV16-detect to enhance clinical decision-making and in avoiding unnecessary surgical procedures. These findings were confirmed in a separate prospective validation cohort.

Wang et al retrospectively measured, ctDNA in saliva $(n=93)$ and plasma $(n=47)$ of 93 patients with head and neck cancer of whom 73 had LAHNC. ctDNA $(n=84)$ and HPV DNA $(n=21)$ was quantified in plasma at a single time point in the majority of patients using personalised PCR and E7 digital PCR, respectively. HPV DNA was detected in plasma in $86 \%(18 / 21)$ of HPV + patients (Wang et al, 2015). However, post-treatment monitoring of HPV DNA was not reported. Dahlstrom et al $(n=114)$, Cao et al $(n=40)$ and Ahn et al $(n=52)$ used E6 and/or E7 PCR for plasma HPV DNA measurement following confirmation of HPV status in tumour (E7 RNA). HPV DNA at baseline was respectively detected in $60 \%, 65 \%$ and $67 \%$ of HPV + patients (Cao et al, 2012; Ahn et al, 2014; Dahlstrom et al, 2015). Mazurek et al based the diagnosis of HPV + LAHNC solely on the presence of plasma HPV DNA quantified using E6/E7 PCR. The detection rate in that study was $14 \%$ (Mazurek et al, 2016). Studies have also attempted to quantify HPV DNA in saliva of HPV patients with LAHNC. However, the detection rate at baseline is between 40 and $55 \%$ and inferior to that of plasma (Ahn et al, 2014; Rettig et al, 2015; Wang et al, 2015). Measuring plasma HPV DNA using targeted deep sequencing (HPV16-detect) with greater coverage of HPV DNA, may have greater sensitivity and specificity compared to other PCR based assays that focus on integrated E6 or E7 (Cao et al, 2012; Dahlstrom et al, 2015; Jeannot et al, 2016; Mazurek et al, 2016) as demonstrated in our study.

Standard-of-care radiological examination is undertaken $10-12$ weeks following CCRT, assuming that disease will have reached maximal response at this time point. However, Huang et al (2013) showed that temporal regression of tumour in lymph nodes in 
$\mathrm{HPV}+$ patients following CCRT might be significantly more prolonged. Biopsies from three patients in our study who had undetectable HPV DNA and persistent ${ }^{18}$ F-FDG uptake and at the primary site at the 12 week time point failed to show residual tumour with subsequent resolution of ${ }^{18} \mathrm{~F}$-FDG uptake, suggesting that some HPV-driven primary tumours exhibit prolonged temporal regression, similar to that seen in lymph node disease. (Figure 2C-E). In addition, plasma HPV DNA levels of can serve as a predictive marker of residual disease in this setting and prevent unnecessary biopsies and the anxiety associated with uncertainty about disease response.

Currently, patients with residual disease (increased ${ }^{18}$ F-FDG uptake; Mehanna et al, 2016) at 10-12 weeks, are considered for salvage surgery for the primary tumour and/or residual lymph nodes. One patient in the test and 3 patients in the validation cohort were deemed to have residual lymph node disease on ${ }^{18} \mathrm{~F}$ FDG PET-CT and underwent ND, without evidence of viable tumour on pathological examination. Plasma HPV DNA was undetectable in all four patients at the time of radiological examination. The randomized phase III PET-NECK study reported a severe surgical complication rate of $20-26 \%$ following ND (Mehanna et al, 2016). In addition, on long-term follow-up, ND following CCRT results in significant fibrosis and reduction in shoulder movement and has an adverse impact on QOL (Shah et al, 2001). Furthermore, HPV + patients are younger and due to the excellent treatment outcomes with the current treatments are expected to carry the burden of treatment related toxicity for life. Over the last few decades, incidence of HPV + LAHNC has significantly increased and is projected to increase by up to $230 \%$ in the near future (Mehanna et al, 2013; Schache et al, 2016). Treatment dilemmas' as a result of 'false positive' ${ }^{18} \mathrm{~F}$ FDG PET-CT will be increasingly common at tumour board meetings. Incorporating measurement of plasma HPV DNA into the management algorithm as a predictor of 'true' residual disease has significant potential to guide treatment decisions and avoid unnecessary surgical procedures and their attendant morbidity.

HPV-driven LAHNC's demonstrate very good response rates to CCRT and only a single patient in our study had residual disease with high levels of HPV DNA. Similarly, HPV DNA was present in samples of small number of patients with residual or recurrent disease in studies by by Cao et al (3 out of 4 patients) and Ahn et al (5 out of 6 patients), where post-treatment plasma samples were available (Cao et al, 2012; Ahn et al, 2014). In the single patient in our study and the study by Cao et al HPV DNA levels were elevated prior to clinically and radiologically detected metastatic disease. In patients with clinical remission under surveillance, more sensitive surveillance strategies using HPV DNA can potentially detect disease relapse before it becomes clinically apparent and can improve the probability of radical salvage therapy.

Our findings require validation in a larger cohort of patients with longer follow-up to help establish the role of HPV16-detect in this setting. Our assay could be used for HPV DNA measurement in other HPV related cancers such as cancers of the cervix, anal canal and penis and pilot studies in patients with cancers at these sites are currently underway at our institution. Clinical trials of treatment de-escalation in HPV-driven tumours are underway and new studies are being planned (Masterson et al, 2014). Such studies require confirmation of HPV as the oncogenic driver, which is currently performed using a combination of $\mathrm{p} 16^{\mathrm{INK} 4 \mathrm{~A}}$ overexpression on IHC and HPV DNA in situ hybridisation on diagnostic tumour blocks, which have a combined sensitivity and specificity of $88 \%$ and $90 \%$, respectively (Schache et al, 2011). Our assay performed on an easily obtained venous sample, with higher specificity and sensitivity, may provide a more practical alternative.

In conclusion, present preliminary results of a novel highly sensitive and specific NGS HPV16-detect assay for detection of
HPV DNA in HPV + patients with non-metastatic LAHNC. In addition, we provide proof of principle that HPV DNA can potentially be used as a predictive biomarker of disease response at the primary site and cervical lymph nodes, following radical treatment for LAHNC, thus avoiding unnecessary surgery.

\section{ACKNOWLEDGEMENTS}

This work was undertaken in The Royal Marsden NHS Foundation Trust and The Institute of Cancer Research. The Royal Marsden NHS Foundation Trust received a proportion of its funding from the NHS Executive; the views expressed in this publication are those of the authors and not necessarily those of the NHS Executive. This work was supported by the National Institute for Health Research Royal Marsden and Institute of Cancer Research Biomedical Research Centre and the Clinical Research Facility BRC grant no. A67. We acknowledge the support of Cancer Research UK Programme Grants C46/A10588 and C7224/A13407. The charity, Oracle Cancer trust funded a part of the project.

\section{CONFLICT OF INTEREST}

The authors declare no conflict of interest.

\section{REFERENCES}

Abgral R, Querellou S, Potard G, Le Roux PY, Le Duc-Pennec A, Marianovski R, Pradier O, Bizais Y, Kraeber-Bodere F, Salaun PY (2009) Does 18F-FDG PET/CT improve the detection of posttreatment recurrence of head and neck squamous cell carcinoma in patients negative for disease on clinical follow-up? J Nucl Med 50(1): 24-29.

Ahn SM, Chan JY, Zhang Z, Wang H, Khan Z, Bishop JA, Westra W, Koch WM, Califano JA (2014) Saliva and plasma quantitative polymerase chain reaction-based detection and surveillance of human papillomavirusrelated head and neck cancer. JAMA Otolaryngol Head Neck Surg 140(9): 846-854.

Bhide S, Clark C, Harrington K, Nutting CM (2007) Intensity modulated radiotherapy improves target coverage and parotid gland sparing when delivering total mucosal irradiation in patients with squamous cell carcinoma of head and neck of unknown primary site. Med Dosim 32(3): 188-195.

Blanchard P, Bourhis J, Lacas B, Posner MR, Vermorken JB, Hernandez JJ, Bourredjem A, Calais G, Paccagnella A, Hitt R, Pignon JP. Meta-Analysis of Chemotherapy in H, Neck Cancer IPCG (2013) Taxane-cisplatinfluorouracil as induction chemotherapy in locally advanced head and neck cancers: an individual patient data meta-analysis of the meta-analysis of chemotherapy in head and neck cancer group. J Clin Oncol 31(23): 2854-2860.

Burk RD, Harari A, Chen Z (2013) Human papillomavirus genome variants. Virology 445(1-2): 232-243.

Cao H, Banh A, Kwok S, Shi X, Wu S, Krakow T, Khong B, Bavan B, Bala R, Pinsky BA, Colevas D, Pourmand N, Koong AC, Kong CS, Le QT (2012) Quantitation of human papillomavirus DNA in plasma of oropharyngeal carcinoma patients. Int J Radiat Oncol Biol Phys 82(3): e351-e358.

Cullen M, Boland JF, Schiffman M, Zhang X, Wentzensen N, Yang Q, Chen Z, Yu K, Mitchell J, Roberson D, Bass S, Burdette L, Machado M, Ravichandran S, Luke B, Machiela MJ, Andersen M, Osentoski M, Laptewicz M, Wacholder S, Feldman A, Raine-Bennett T, Lorey T, Castle PE, Yeager M, Burk RD, Mirabello L (2015) Deep sequencing of HPV16 genomes: A new high-throughput tool for exploring the carcinogenicity and natural history of HPV16 infection. Papillomavirus Res 1: 3-11.

Dahlstrom KR, Li G, Hussey CS, Vo JT, Wei Q, Zhao C, Sturgis EM (2015) Circulating human papillomavirus DNA as a marker for disease extent and recurrence among patients with oropharyngeal cancer. Cancer 121(19): 3455-3464. 
Dillon MT, Harrington KJ (2015) Human papillomavirus-negative pharyngeal cancer. J Clin Oncol 33(29): 3251-3261.

Garcia-Murillas I, Schiavon G, Weigelt B, Ng C, Hrebien S, Cutts RJ, Cheang M, Osin P, Nerurkar A, Kozarewa I, Garrido JA, Dowsett M, Reis-Filho JS, Smith IE, Turner NC (2015) Mutation tracking in circulating tumor DNA predicts relapse in early breast cancer. Sci Transl Med 7(302): 302ra133.

Hamoir M, Ferlito A, Schmitz S, Hanin FX, Thariat J, Weynand B, Machiels JP, Gregoire V, Robbins KT, Silver CE, Strojan P, Rinaldo A, Corry J, Takes RP (2012) The role of neck dissection in the setting of chemoradiation therapy for head and neck squamous cell carcinoma with advanced neck disease. Oral Oncol 48(3): 203-210.

Hopman AH, Smedts F, Dignef W, Ummelen M, Sonke G, Mravunac M, Vooijs GP, Speel EJ, Ramaekers FC (2004) Transition of high-grade cervical intraepithelial neoplasia to micro-invasive carcinoma is characterized by integration of HPV 16/18 and numerical chromosome abnormalities. J Pathol 202(1): 23-33.

Huang SH, O'Sullivan B, Xu W, Zhao H, Chen DD, Ringash J, Hope A, Razak A, Gilbert R, Irish J, Kim J, Dawson LA, Bayley A, Cho BC, Goldstein D, Gullane P, Yu E, Perez-Ordonez B, Weinreb I, Waldron J (2013) Temporal nodal regression and regional control after primary radiation therapy for N2-N3 head-and-neck cancer stratified by HPV status. Int J Radiat Oncol Biol Phys 87(5): 1078-1085.

Jeannot E, Becette V, Campitelli M, Calméjane M-A, Lappartient E, Ruff E, Saada S, Holmes A, Bellet D, Sastre-Garau X (2016) Circulating human papillomavirus DNA detected using droplet digital PCR in the serum of patients diagnosed with early stage human papillomavirus-associated invasive carcinoma. J Pathol Clin Res 2(4): 201-209.

Lamarcq L, Deeds J, Ginzinger D, Perry J, Padmanabha S, Smith-McCune K (2002) Measurements of human papillomavirus transcripts by real time quantitative reverse transcription-polymerase chain reaction in samples collected for cervical cancer screening. J Mol Diagn 4(2): 97-102.

Li H, Handsaker B, Wysoker A, Fennell T, Ruan J, Homer N, Marth G, Abecasis G, Durbin R. Genome Project Data Processing S (2009) The Sequence Alignment/Map format and SAMtools. Bioinformatics 25(16): 2078-2079.

Masterson L, Moualed D, Liu ZW, Howard JE, Dwivedi RC, Tysome JR, Benson R, Sterling JC, Sudhoff H, Jani P, Goon PK (2014) De-escalation treatment protocols for human papillomavirus-associated oropharyngeal squamous cell carcinoma: a systematic review and meta-analysis of current clinical trials. Eur J Cancer 50(15): 2636-2648.

Mazurek AM, Rutkowski T, Fiszer-Kierzkowska A, Malusecka E, Skladowski K (2016) Assessment of the total cfDNA and HPV16/18 detection in plasma samples of head and neck squamous cell carcinoma patients. Oral Oncol 54: 36-41.

Mehanna H, Beech T, Nicholson T, El-Hariry I, McConkey C, Paleri V, Roberts S (2013) Prevalence of human papillomavirus in oropharyngeal and nonoropharyngeal head and neck cancer-systematic review and metaanalysis of trends by time and region. Head Neck 35(5): 747-755.
Mehanna H, Wong WL, McConkey CC, Rahman JK, Robinson M, Hartley AG, Nutting C, Powell N, Al-Booz H, Robinson M, Junor E, Rizwanullah M, von Zeidler SV, Wieshmann H, Hulme C, Smith AF, Hall P, Dunn J. Group P-NTM (2016) PET-CT surveillance versus neck dissection in advanced head and neck cancer. N Engl J Med 374(15): 1444-1454.

Quinlan AR, Hall IM (2010) BEDTools: a flexible suite of utilities for comparing genomic features. Bioinformatics 26(6): 841-842.

Rettig EM, Wentz A, Posner MR, Gross ND, Haddad RI, Gillison ML, Fakhry C, Quon H, Sikora AG, Stott WJ, Lorch JH, Gourin CG, Guo Y, Xiao W, Miles BA, Richmon JD, Andersen PE, Misiukiewicz KJ, Chung CH, Gerber JE, Rajan SD, D'Souza G (2015) Prognostic implication of persistent human Papillomavirus type 16 DNA detection in oral rinses for human papillomavirus-related oropharyngeal carcinoma. JAMA Oncol 1(7): 907-915.

Schache AG, Liloglou T, Risk JM, Filia A, Jones TM, Sheard J, Woolgar JA, Helliwell TR, Triantafyllou A, Robinson M, Sloan P, Harvey-Woodworth C, Sisson D, Shaw RJ (2011) Evaluation of human papilloma virus diagnostic testing in oropharyngeal squamous cell carcinoma: sensitivity, specificity, and prognostic discrimination. Clin Cancer Res 17(19): 6262-6271.

Schache AG, Powell NG, Cuschieri KS, Robinson M, Leary S, Mehanna H, Rapozo D, Long A, Cubie H, Junor E, Monaghan H, Harrington KJ, Nutting CM, Schick U, Lau AS, Upile N, Sheard J, Brougham K, West CM, Oguejiofor K, Thomas S, Ness AR, Pring M, Thomas GJ, King EV, McCance DJ, James JA, Moran M, Sloan P, Shaw RJ, Evans M, Jones TM (2016) HPV-related oropharynx cancer in the United Kingdom: an evolution in the understanding of disease etiology. Cancer Res 76(22): 6598-6606.

Shah S, Har-El G, Rosenfeld RM (2001) Short-term and long-term quality of life after neck dissection. Head Neck 23(11): 954-961.

Wang Y, Springer S, Mulvey CL, Silliman N, Schaefer J, Sausen M, James N, Rettig EM, Guo T, Pickering CR, Bishop JA, Chung CH, Califano JA, Eisele DW, Fakhry C, Gourin CG, Ha PK, Kang H, Kiess A, Koch WM, Myers JN, Quon H, Richmon JD, Sidransky D, Tufano RP, Westra WH, Bettegowda C, Diaz Jr. LA, Papadopoulos N, Kinzler KW, Vogelstein B, Agrawal N (2015) Detection of somatic mutations and HPV in the saliva and plasma of patients with head and neck squamous cell carcinomas. Sci Transl Med 7(293): 293ra104.

Wilkins AC, Rosenfelder N, Schick U, Gupta S, Thway K, Nutting CM, Harrington KJ, Newbold K, Bhide SA (2013) Equivalence of cisplatin and carboplatin-based chemoradiation for locally advanced squamous cell carcinoma of the head and neck: a matched-pair analysis. Oral Oncol 49(6): 615-619.

This work is published under the standard license to publish agreement. After 12 months the work will become freely available and the license terms will switch to a Creative Commons AttributionNonCommercial-Share Alike 4.0 Unported License.

Supplementary Information accompanies this paper on British Journal of Cancer website (http://www.nature.com/bjc) 